\title{
Zur Methodik der Lipoprotein-Elektrophorese auf Celluloseacetat-Folie im Routinelaboratorium
}

\author{
Von G. B. ZIEGLER \\ Klin.-chem. Zentrallaboratorium (Chefarzt: Dr. F. Wosegien) des Kreiskrankenbauses Ludwigsburg
}

(Eingegangen am 30. Juni/24. August 1972)

Eine einfache, für das Routinelaboratorium geeignete Methode der Lipoprotein-Elektrophorese wird beschrieben. Sie zeichnet sich durch scharfe Trennung der Fraktionen und durch eine intensive, stabile Färbung aus. Aussagekraft und Fehlerquellen der Methode werden diskutiert.

\section{Electropboresis of lipoproteins on cellulose acetate in the routine laboratory}

A simple method is described for the electrophoresis of lipoproteins, which is suitable for the routine laboratory. It is characterized by a sharp separation and an intense, stable staining of the fractions. The diagnostic value and the sources of error in the method are discussed.

In Abwandlung der von KLEMENS (1) modifizierten Lipoproteinelektrophorese auf Celluloseacetatfolien nach CHIN (2) haben wir Testbedingungen erarbeitet, die sich wegen des geringen Aufwands zur Verwendung im klinisch-chemischen Routinelaboratorium eignen. Eine densitometrische Auswertung ist möglich.

\section{Material und Methodik}

Apparatur zur Mikrozonenelektrophorese auf Celluloseacetatfolien (z. B. Fa. Boskamp).

Densitometer zur Auswertung von Mikrozonenelektrophoresen (z. B. Chromoscan der Fa. Joyce, Loebl \& Co. Ltd.).

Heizplatte mit Magnetrührer.

Celluloseacetatfolien (Schleicher und Schüll).

Michaelispuffer $0,06 \mathrm{~mol} / \mathrm{l} \mathrm{pH} \mathrm{8,6.}$

Ölrot 0 (Serva), Methanol p. a., Eisessig, Natriumhypochloritlösung 12,5\% (technisch).

Das nach mindestens 12-stündiger Nahrungskarenz gewonnene Patientenserum wird möglichst frisch zur Elektrophorese eingesetzt. Kleine Probenmengen werden für evtl. notwendige weitere Untersuchungen bei $-78^{\circ} \mathrm{C}$ (Methanol/Trockeneis) eingefroren und bei $-18^{\circ} \mathrm{C}$ gelagert. Nach dem Auftauen werden sie nicht wieder eingefroren. Stets werden Serum-Cholesterin und SerumTriglyceride mitbestimmt. Die Verwendung von Heparinplasma bringt keine Vorteile.

\section{Elektroplsorese}

Die Vorbereitung der Folien erfolgt wie bei der Eiweiß-Mikrozonenelektrophorese üblich. Zum Auftragen der Probe sind wir nach vorübergehendem Gebrauch von Mikrokapillaren (1) dazu übergegangen, die Schmalkante eines Objektträgers durch Eintauchen in einen Tropfen Serum gleichmäßig zu benetzen und kurz kathodenwärts auf die Folie aufzulegen. Hierbei werden 3-5 $\mu \mathrm{l}$ Serum in einer scharf begrenzten Startlinie aufgetragen. Nach vollständigem Eintrocknen der Probe in die Folie erfolgt die Trennung bei $250 \mathrm{~V}$. Die Dauer ist abhängig von der erreichten Trennstrecke (mindestens $3 \mathrm{~cm}$, kontrollierbar an der deutlichen Albuminfront), etwa $40 \mathrm{~min}$. Danach werden die Folien feucht in das Farbbad eingelegt und $12-24 \mathrm{~h}$ bei $37^{\circ} \mathrm{C}$ gefärbt.

Farbbad und Transparenzuerfabren

$\mathrm{Zu} 160 \mathrm{ml}$ siedendem Methanol werden $0,5 \mathrm{~g}$ Olrot 0 gegeben und 5 min unter ständigem Rühren aufgekocht. Auf einen Rück- flußkühler kann verzichtet werden. Langsam wird die ständig siedende Lösung mit Methanol/Wasser $(11+11)$ auf $300 \mathrm{ml}$ aufgefüllt, wobei ein Ausfallen des Ölrot vermieden werden soll. Die Lösung soll zum Schluß tiefrot und nur gering getrübt sein. Der Wassergehalt soll $30 \%$ nicht übersteigen, da dies die Löslichkeit des Ölrot stark vermindert, soll aber nicht unter $20 \%$ bleiben, da dann Lipide aus der Folie herausgelöst werden. Nach dem Abkühlen wird die Farblösung ins Färbegefä $B$ dekantiert und abgedeckt bei $37^{\circ} \mathrm{C}$ aufbewahrt. Sie ist etwa 7 Tage gebrauchsfähig.

$\mathrm{Zu} 80 \mathrm{ml}$ 5proz. Essigsäure werden $5 \mathrm{ml} \mathrm{12,5proz.} \mathrm{Natrium-}$ hypochloritlösung gegeben (Abzug). Die Folien werden einzeln dem Farbbad entnommen, durch leichtes Darüberwischen von aufgelagertem Ölrotniederschlag befreit und einzeln 15-20 s in der Natriumhypochloritlösung entfärbt. Der Untergrund sollte nur leicht rötlich, die Lipoproteinfraktionen tiefrot gefärbt sein. Sollte der Farbeffekt nocht, nicht ausreichend sein, kann der Färbevorgang nach kurzem Spülen der Folie in Wasser fortgesetzt werden. Vor der Transparenzbehandlung werdeñ die Folien $10 \mathrm{~min}$ in Wasser gespült. Sie werden dann für $2 \mathrm{~s}$ ins Transparenzbad (Eisessig/Methanol $900 \mathrm{ml}+100 \mathrm{ml}$ ) eingetaucht und sofort auf einen Objektträger aufgezogen. Gasblasen werden wie üblich mit einem Glasstab oder dgl. entfernt. Werden die Folien vor der Transparenzbehandlung aufgezogen, dann bilden sich im Transparenzbad Gasblasen unter der Folie, die nichț entfernt werden können. Unter Warmluft werden die transparenten Folien getrocknet. Falls notwendig, kann dann die Transparenzbehandlung jetzt an den aufgezogenen Folien wiederholt werden.

\section{Ergebnisse und Diskussion}

Mit der geschilderten Methode lassen sich Lipoproteinelektrophoresen mit guter Reproduzierbarkeit durchführen. Bei mehrfacher $(n=30)$ Auftrennung eines Normalserums betrug die Standardabweichung für die $\alpha$-Lipoproteine $2,3 \%$, für die prä- $\beta$-Lipoproteine $0,9 \%$ und für die $\beta$-Lipoproteine 3,0\% der Gesamtextinktion. KLEMENS (1) gab Streubreiten von 3,6\%,2,9\% und $3,0 \%$ bei mehrfacher Auftrennung eines Normalserums an.

Die Ergebnisse der Lipoproteinelektrophorese auf Celluloseacetat sind denen der Auftrennung im Agarosegel vergleichbar $(1,3,5,6)$, die Trennschärfe ist 


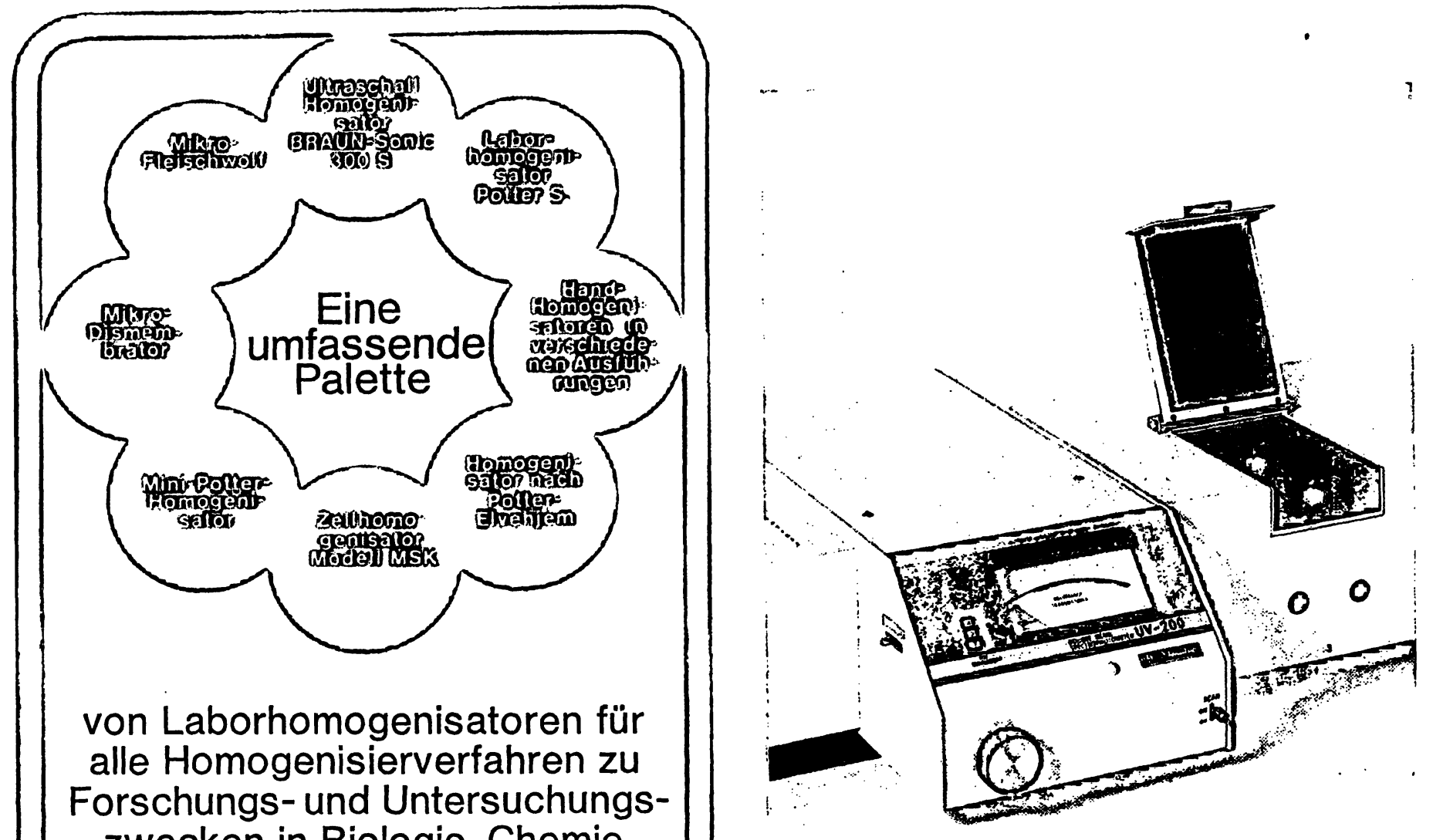

zwecken in Biologie, Chemie,

Physik, Botanik, Zoologie,

Pharmakologie, Physiologie und anderen Disziplinen. Jedes Gerät ist für ein spezielles Verfahren besonders geeignet.

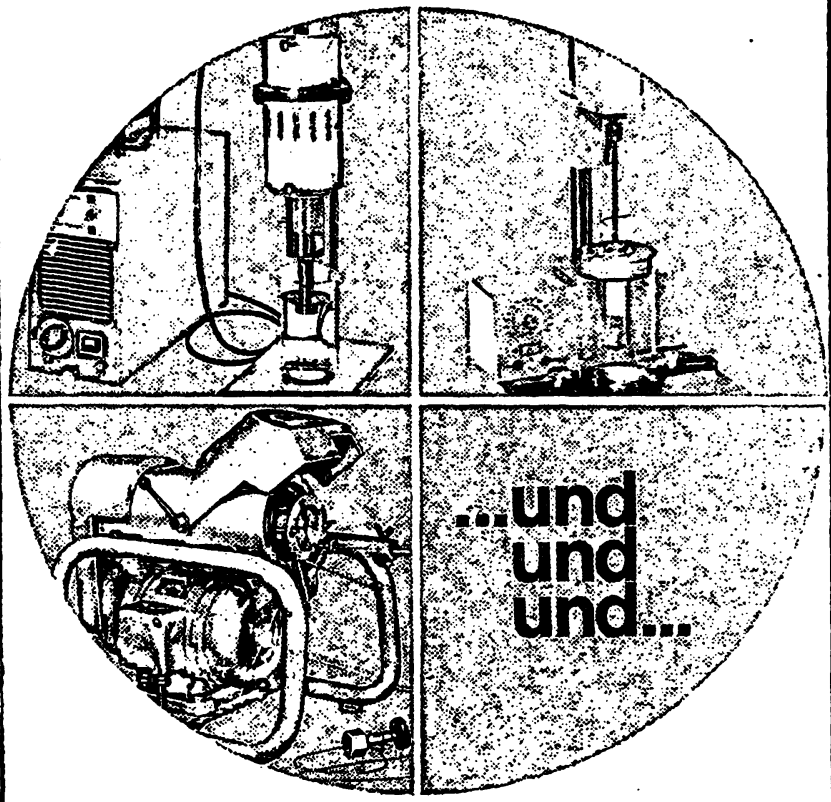

\section{B. Braun Melsungen Aktiengesellschaft} Medizin-u.Labortechnik

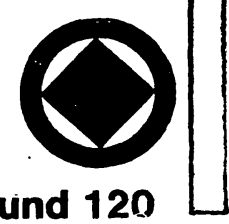

Shimadzu-UV-200 Spektralphotometer

Registrierendes Zweistrahlphotometer hervorragender Qualität. Ein neuartiger Strahlenteiler garantiert hohe analytische Genauigkeit. Spektralbereich $195 \mathrm{~nm}$ bis $850 \mathrm{~nm}$. Czerny-Turner-Gittermonochromator. Einfache Bedienung durch automatische $0 \%$ - und $100 \%$ Korrektur. Äußerst exakte $100 \%$ Linie. Ein modernes Gerät zu sehr günstigem Preis.

\section{Autorisierte Vertretung:}

\section{Colora Messtechnik GmbH} 7073 Lorch/Württ. 1, Postfach 5 T (07172) 6041, FS 07-248886

Technische Büros (Verkauf und Kundendienst): 1000 Berlin 30, Ansbacher Straße 17-19, T 244090 2000 Hamburg 19, Osterstraße 63, T 491 10 34, FS 02-12 947 3000 Hannover, An der. Tiefenriede 45, T 884500

4000 Düsseldorf 1, Kronprinzenstr. 62, T 320164 , FS $08-587253$ 6000 Frankfurt a.M., Röderbergweg 4-6, T 446031, FS 04-11216 8000 München 19, Dachauer Straße 175, T 193858

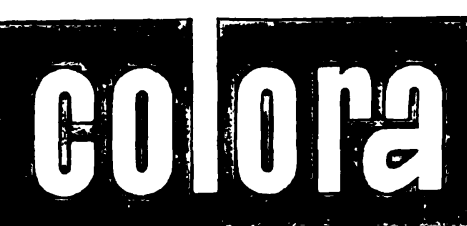




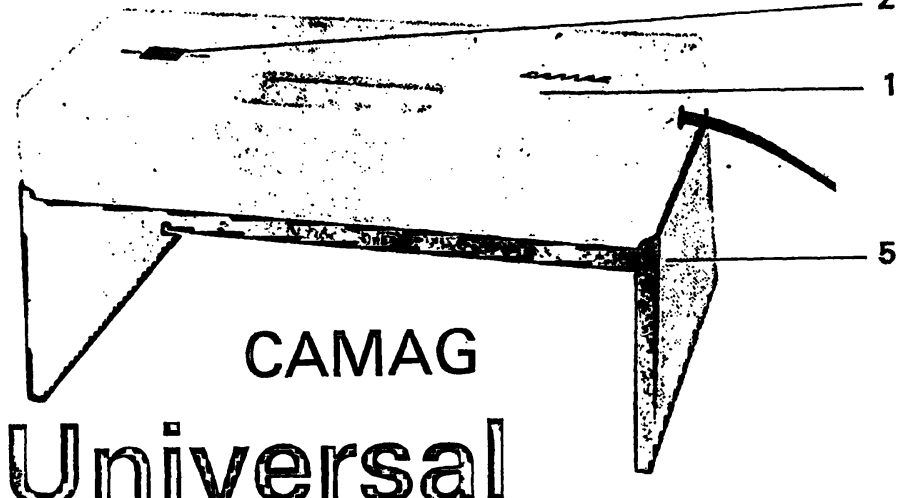

UV-Lampe

für die DünnschichtChromatographie

langwelliges UV $366 \mathrm{~nm}$ kurzwelliges UV $254 \mathrm{~nm}$
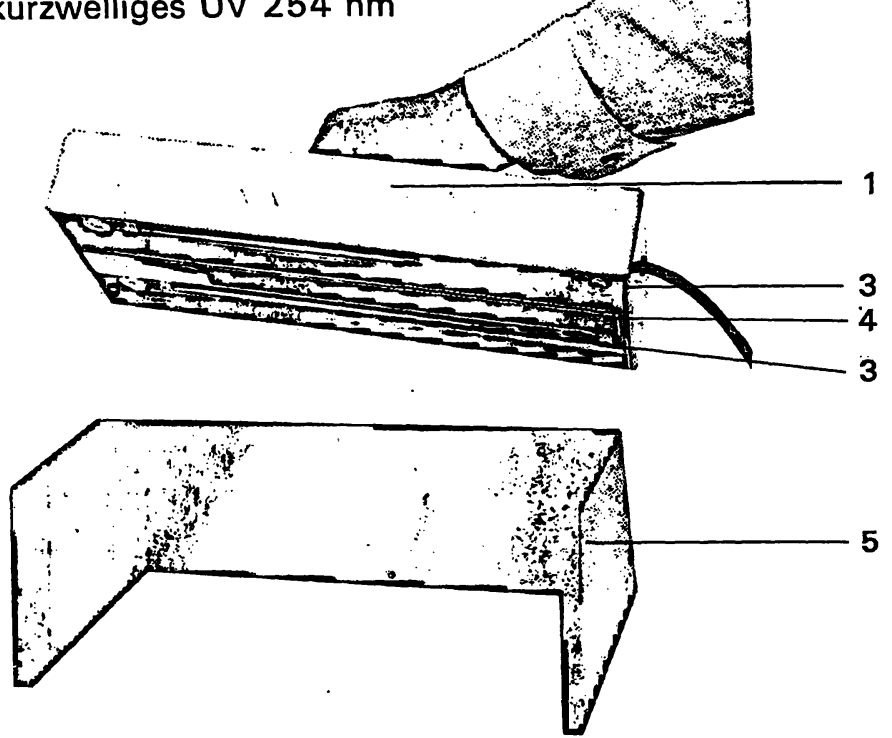

1 formschönes, handliches Gehäuse aus schlagfestem Kunststoff

2 Kombinationsschalter ein - aus - $366 \mathrm{~nm}-254 \mathrm{~nm}$

3 zwei Röhren für das langwellige UV ergeben eine grosse Leuchtdichte

4 abnehmbarer UV-Filter mit geometrisch optimal angeordnetem Fenster für das kurzwellige UV

5 Stativ, schirmt Fremdlicht auf drei Seiten wirksam ab
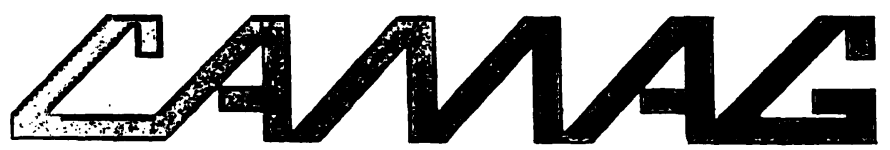

Führend in

Dünnschicht-Chromatographie Dünnschicht-Elektrophorese Hochspannungs-Elektrophorese
4132 Muttenz/Schweiz Homburgerstrasse 24 Tel. (061) 531430
1000 Berlin 41

Bismarckstrasse 27-29 Tel. (0311) 7915091

\section{Walter de Gruyter Berlin.New York}

Neuerscheinungen

\section{Hilde Götz}

\section{Immunologische Plasmaprotein-Diagnostik}

Oktav. XII, 256 Seiten. Mit 106 Abbildungen. 1973. Gebunden DM 96,-

ISBN 3110037726

Das Buch vermittelt Methodik und klinischen Anwendungsbereich der wichtigsten immunologișchen Untersuchungstechniken zur Differenzierung menschlicher Plasmaproteine. Es werden die Immunelektrophorese (Grabar und Williams bzw. Scheidegger) mit reichlichem Anschauungsmaterial und Inferpretationshilfen, die zweidimensionale Doppel-Immundiffusion (Ouchterlony) sowie die einfache, radiale Immundiffusion im Agargel (Mancini und Mitarb.) dargelegt.

\section{Edward Muntwyler}

\section{Elektrolytstoffwechsel und Säure-Basen-Gleichgewicht}

Übersetzt aus dem Englischen von

Wolf-Dieter Thomitzek

Klein-Oktav. 260 Seiten. Mit 33 Abbildungen. 1973. Kartoniert DM 14,80 ISBN 3110039508

(Sammlung Göschen Band 7137)

In dẹn einführenden Abschnitten über den Wasserund Elektrolythaushalt werden neben den Grundbegriffen Fragen der Verteilung in den Körperkompartimenten, die exkretorische Nierenfunktion und pathologische Abweichungen des Elektrolythaushaltes abgehandelt. Es folgt eine Überblick über den Mechanismus der Aufrechterhaltung des SäureBasen-Gleichgewichts unter physiologischen und pathologischen Bedingungen und über die Mitwirkung der Lunge, der Nieren und anderer Organe. Fragen der klinischen Diagnostik werden ebenso klar dargestellt wie die Grundzüge einer rationellen Therapie pathologischer Zustände.

For USA and Canado:

Please send all orders to Walter de Gruyter Inc 162 Fifth Avenue, New York, N.Y. 10010 Tel. (212) 255-0808 

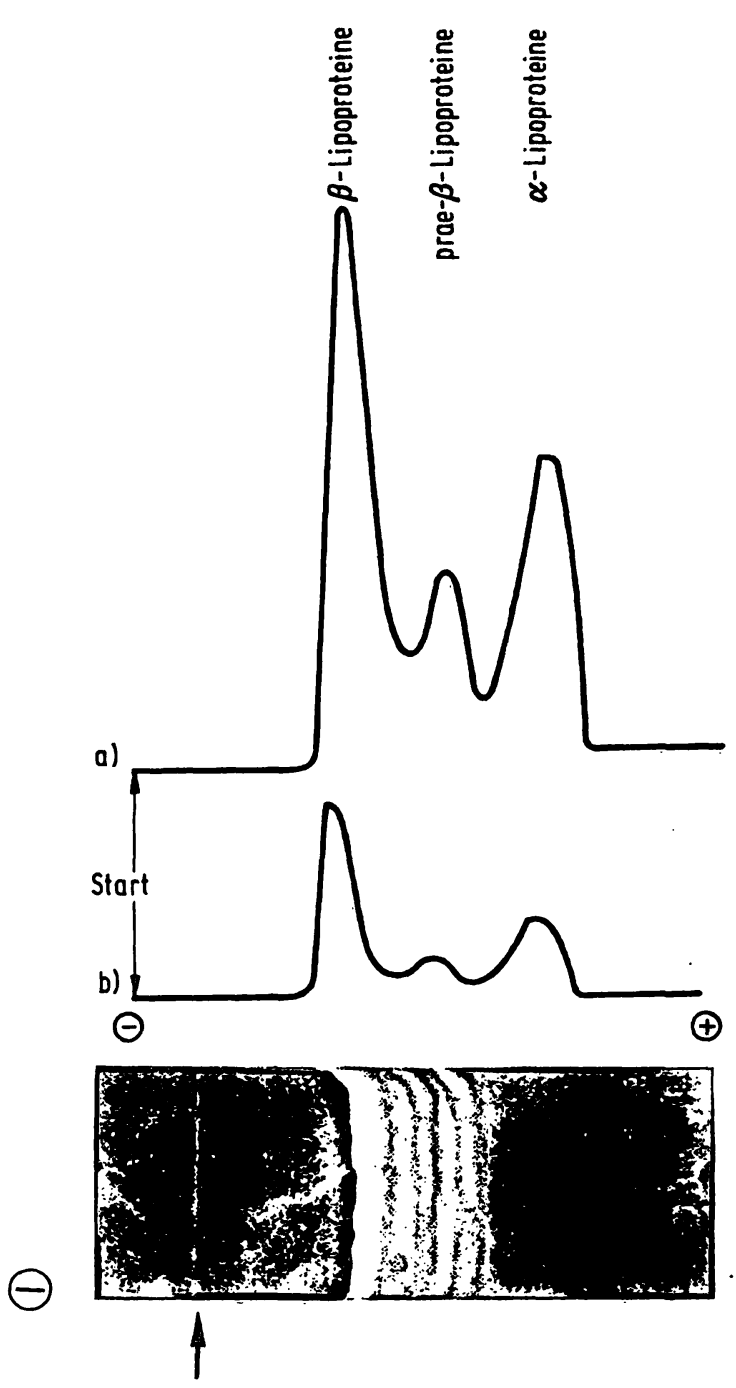

Abb. 1

Lipoprotein-Elektrophorese eines Normalserums ( $\alpha$-Lipoproteine $31 \%$, prä- $\beta$-Lipoproteine $14 \%$, $\beta$-Lipoproteine $55 \%$ ). Bei sonst identischen Methode $(1,5 \mathrm{~g}$ Örot/l), b) nach der Vorschrift von KLEMENS (I) $(0,5 \mathrm{~g}$ Olrot $/ 1)$

aber durch Verwendung von Celluloseacetat und nicht zuletzt durch die geringe eingesetzte Probenmenge auch ohne Albuminzusatz größer (vgl. 1, 4-8). Die Intensität der Färbung ist abhängig von der Konzentration des gelösten Ölrot, weshalb wir 1,5 g Ölrot/1 verwenden (vgl. Abb. 1). Die F̈ärbung ist sowohl im Entfärbe- als auch im Transparenzbad stabil (1). Erst bei einer Verweildauer der Folien im Transparenzbad über $7 \mathrm{~s}$, die normalerweise nicht annähernd erreicht wird, stellten wir eine Verschiebung der einzelnen Anteile an der
Gesamtextinktion zugunsten der prä- $\beta$-Lipoproteine um $\max .6 \%$ auf Kosten der $\beta$-Lipoproteine fest. Der Anteil $\operatorname{der} \alpha$-Lipoproteine blieb konstant.

$\mathrm{Da}$ die prozentualen Anteile der einzelnen Lipidkomponenten in den Lipoproteinen als konstant angenommen werden kann $(10,11,12)$, steht einer densitometrischen (semi-)quantitativen Auswertung nichts im Wege.

Hyperlipoproteinaemien vom Verteilungsmuster Typ I, IIa, IIb, IV und V nach FREDRICKson (3) können meist sicher diagnostiziert werden (vgl. Tab. 1. u. 2). Die mit der Methode erhaltenen Normalwerte sind aus Tabelle 1 ersichtlich (vgl. 1. c. (1), (10)). Laufende Kontrolluntersuchungen dienen der Beurteilung des Therapieerfolgs.

Erhebt sich der Verdacht auf das Vorliegen eines Verteilungsmusters vom Typ III, läßt sich häufig durch Veränderung der Elektrophoresebedingungen (Puffer: Veronal/Veronal-Na/Ca-Lactat $16,9 \mathrm{~g} / 43,8 \mathrm{~g}, 1,9 \mathrm{~g}$, dest. Wasser $5000 \mathrm{ml} ; 0,05 \mathrm{~mol} / \mathrm{l}$, pH 8,6; Trennzeit $60 \mathrm{~min}$ bei einer Trennstrecke von $5 \mathrm{~cm}$ ) eine Abtrennung der prä- $\beta$-Fraktion von den $\beta$-Lipoproteinen erreichen. Andernfalls sind entsprechende Spezialuntersuchungen notwendig. (Ultrazentrifugation, immunologischer Nachweis von LP-X) $(3,13,14)$.

Die stets in geringer Konzentration im Albuminbereich nachweisbaren freien Fettsäuren, die besonders nach längerer Lagerung über $4^{\circ} \mathrm{C}$ infolge der Serum-LipaseAktivität vermehrt sind, werden bei der densitometrischen Auswertung nicht mit berücksichtigt. Mehrfaches Einfrieren und Wiederauftauen der Proben führt durch Molekülcrackung zu einer Vermehrung von Lipoproteinmolekül-Bruchstücken, die in der nachfolgenden Elektrophorese ein Tailing verursachen bzw. am Start liegen bleiben und so das Vorliegen von Chylomikronen vortäuschen. Zentrifugationsversuche (Sorvall high speed Zentrifuge RC2B mit $50000 \mathrm{~g}$ bei $0-4^{\circ} \mathrm{C}$, Dauer $1-6 \mathrm{~h}$ ) an mehrmals bei $-18^{\circ} \mathrm{C}$ eingefrorenen und wieder aufgetauten Seren (Normalseren sowie Verteilungsmuster II und IV) ergaben eine deutliche Vermehrung von an der Oberfläche flottierenden Lipiden, die sich elektrophoretisch wie Chylomikronen verhielten. Mehrfach bei $-78^{\circ} \mathrm{C}$ eingefrorene Seren zeigten diese Veränderungen nicht. Dennoch gingen wir dazu über, einmal aufgetaute Seren nicht wieder einzufrieren, sondern sofort $z u$ verwenden und danach zu verwerfen.

Tab. 1

Lipoprotein-Verteilung (Mittelwerte und Standardabweichung) von 116 Seren aus einem vorwiegend kardiologischen Krankengut

\begin{tabular}{|c|c|c|c|c|c|c|c|c|c|c|}
\hline \multirow[t]{2}{*}{. } & \multirow{2}{*}{$\begin{array}{c}\begin{array}{c}\text { Verteilungs- } \\
\text { muster }\end{array} \\
\text { Normal }\end{array}$} & \multirow{2}{*}{$\frac{n}{35}$} & \multicolumn{2}{|c|}{$\begin{array}{c}\alpha \text {-Lipoproteine } \\
\overline{\mathbf{x}}(\text { rel. } \%) \text { s }\end{array}$} & \multicolumn{2}{|c|}{ prä- $\beta$-Lipoproteine } & \multicolumn{2}{|c|}{$\beta$-Lipoproteine } & \multicolumn{2}{|c|}{ Chylomikronen } \\
\hline & & & 28,3 & 4,5 & 17,3 & 3,7 & 54,4 & 5,6 & 0 & 0 \\
\hline & Typ II a & 17 & 9,5 & 5,5 & 11,2 & 6,6 & 79,4 & 8,8 & 0 & 0 \\
\hline & $\begin{array}{l}\text { Typ IIb } \\
\text { Verdacht }\end{array}$ & 13 & 9,4 & 5,3 & 25,8 & 3,2 & 67,0 & 4,5 & 0 & 0 \\
\hline & auf Typ III & 6 & 5,0 & 3,3 & $86,0^{*}$ & $14,7^{*}$ & & & 10,0 & 14,4 \\
\hline & Typ IV & 33 & $16 ; 4$ & 6,8 & 41,2 & 14,7 & 42,7 & 11,6 & 0 & 0 \\
\hline & Typ V & 12 & 19,7 & 6,9 & 32,3 & 9,5 & 30,2 & 6,3 & 14,2 & 8,3 \\
\hline
\end{tabular}

* $\overline{\mathrm{x}}$ sowie s beziehen sich auf prä- $\beta$ und $\beta$-Lipoproteine 
Tab. 2

Lipidstaten von 15 Patienten

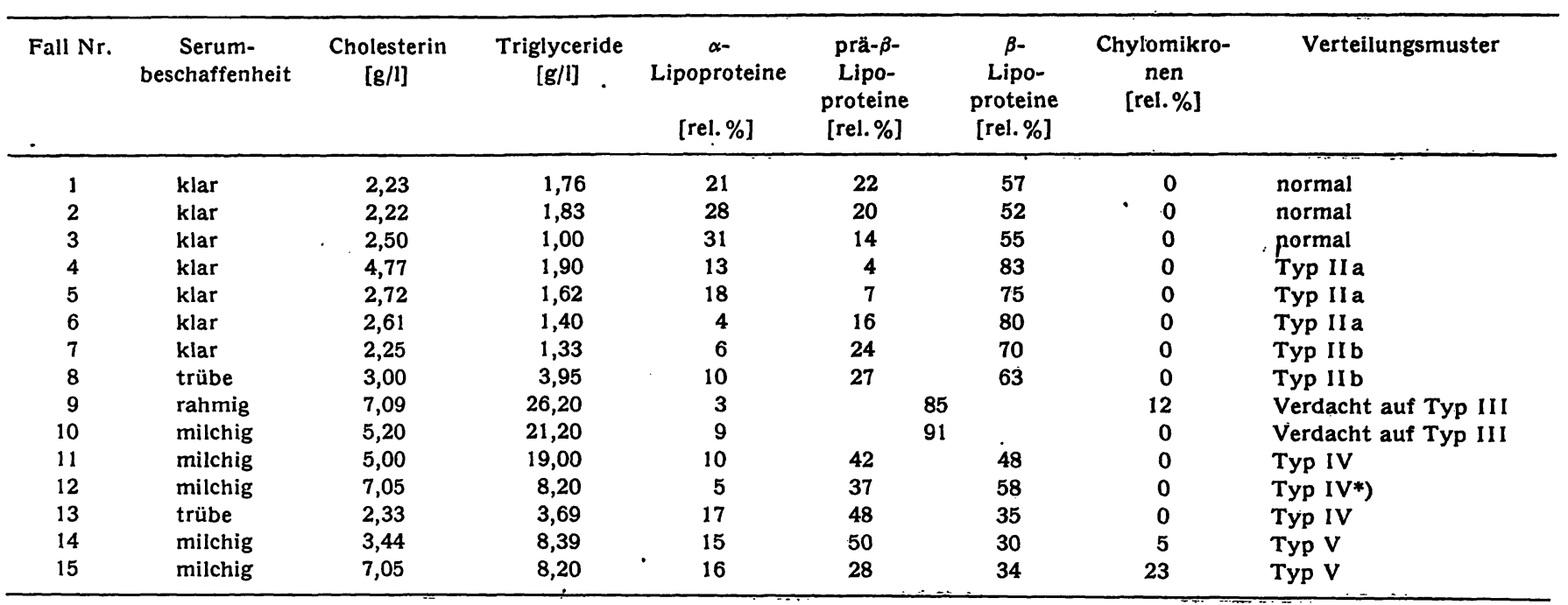

*) Die Trennung der prä- $\beta$-Lipoproteine von den $\beta$-Lipoproteinen erfolgte erst bei Elektrophorese im modifizierten Puffersystem

\section{Literatur}

1. Klemens, U. H. \& Schmalbeck, J. (1969), diese Z. 7, 540 bis 546. - 2. Chin, H. P. \& Blankenhorn, D. H. (1968), Clin. Chim. Acta 20,305-314. - 3. Fredrickson, D. S. (1971), in Hyperlipidaemien (Berg, G. Hrsg.) 1. Aufl. Georg Thieme Verlag Stuttgart, S. 1-18. - 4. SchönBroN, W. et al. in 1. c. (3) S. 28 , 37. - 5. Grabner, W. et al. in 1. c. (3) S. 45-52. - 6. KLEMENS, U. H. in 1. c. (3) S. 53-57. - 7. GRETEN, H. \& SeIDEL, D. in 1. c. (3) S. 58-63. - 8. Huth, K. (1969), Diagnostik 2, 413-416. 9. Wissenschaftl. Tabellen GeIGY (1968), 7. Aufl. S. 596-600. -
10. Grabner, W. et al. (1970), Clin. Chim. Acta 28, 299-310. 11. Klemerns, U. H. et al. (1972), Klin. Wochenschr. 50, 139-148. 12. SEIDEL, D. (1971), in Fettstoffwechselstörungen (Schettler, G. Hrsg.). 1. Aufl., Georg Thieme Verlag Stuttgart, S. 24-47. 13. Seidel, D. \& Greten, H. (1970), Clin. Chim. Acta 30, 31-36. 14. SerDel, D. in l. c. (3) S. 19-27. - 15. Schwañ̃t, P. (1971), Internist 11, 481-487. - 16. ZölLNER, N. et al. (1969), diese Z. 7, 525-529. - 17. Klemens, U. H. \& Schmalbeck, J. (1970), diese Z. 8, 162-165; 166-169.

Dr. G. B. Ziegler 714 Ludwigsburg Posilipostr. 49 